\title{
DE NUEVO SOBRE ATAECINA Y TUROBRIGA. EXPLORACIONES DEL AÑO 1900 EN LAS TORRECILLAS (ALCUÉSCAR, CÁCERES)
}

\author{
POR \\ JUAN MANUEL ABASCAL \\ Universidad de Alicante
}

\section{RESUMEN}

Un manuscrito conservado en la Real Academia de la Historia contiene los resultados de una prospección arqueológica llevada a cabo en 1900 en el paraje de Las Torrecillas en Alcuéscar (Cáceres), el lugar del que pudieron extraerse las inscripciones halladas en Santa Lucía del Trampal. El paraje de Las Torrecillas podría coincidir con la ubicación de Turobriga.

\section{SUMMARY}

A manuscript in the Real Academia de la Historia (Madrid) contains the data on an archaeological prospection carried out in 1900 in Las Torrecillas (Alcuéscar, prov. Cáceres). The inscriptions found in Sta. Lucía probably come from Las Torrecillas, site that could be identified with Turobriga.

El 9 de abril de 1900 la Real Academia de la Historia fue informada por sus Correspondientes en Cáceres de que en el término de Alcuéscar, y como consecuencia de la realización de trabajos agrícolas en el paraje de Las Torrecillas, había aparecido un gran número de objetos romanos y un subterráneo con estructuras de ladrillo en su interior. El informe, manuscrito y extenso, iba acompañado de una carta de Joaquín Santos Ecay, Presidente de la Comisión de Monumentos de Cáceres, y en él se daba cuenta de los hallazgos así como de las excavaciones que se habían hecho tras su conocimiento. Dos meses más tarde, el 28 de junio del mismo año, la citada Comisión remitió la memoria de los trabajos realizados, así como una cajita conteniendo huesos hallados en el lugar. Esta memoria final venía ya firmada por Juan Sanguino y Michel, a la sazón Correspondiente de la Academia, e instigador de los trabajos llevados a cabo en su calidad de Secretario de la citada Comisión de Monumentos cacereña; lleva por título «Memoria sobre los descubrimientos hechos en Alcuéscar. 1900» ${ }^{1}$.

Toda la documentación sobre el particular se conserva en la Biblioteca de la Real Academia de la Historia, Sección de Antigüedades, legajo 9-7848-22; allí lo vimos en abril de
La Real Academia de la Historia trató el asunto en sus reuniones del 14 de abril de 1900 (en donde se dio cuenta del descubrimiento) y 30 de junio (sobre las fotos recibidas), hasta que el 4 de julio de 1900 comunicó a Santos Ecay la publicación de los resultados de sus pesquisas en el Boletín de la Institución ${ }^{2}$. Varios años más tarde J. Sanguino retomó la información aún inédita contenida en la «Memoria» de 1900 y publicó un estudio más amplio que contenía ya la descripción pormenorizada de los hallazgos ${ }^{3}$. A partir de la información contenida en esos documentos, se puede confeccionar el siguiente relato de los hechos:

A finales de marzo de 1900, un periódico cacereño - El Norte de Extremadura - publicó una carta de D. Rafael García-Plata de Osuna comunicando la aparición cerca de Alcuéscar de diversos objetos arqueológicos. A la vista de esas noticias, la Comisión Provincial de Monumentos, presidida entonces por D. Joaquín Santos Ecay, envió a tres de sus miembros para que realizaran una inspección sobre el terreno a fin de evaluar la importancia de los descubrimientos; integraban la delegación $\mathrm{D}$. Daniel Berjano, el arquitecto provincial D. Emilio $\mathrm{M}^{\mathrm{a}}$ Rodríguez y D. Juan Sanguino Michel, que por entonces residía temporalmente en la propia localidad de Alcuéscar. Juntos se trasladaron el día 4 de abril a la finca de Las Torrecillas, de donde procedían los objetos hallados, y realizaron una primera evaluación de los descubrimientos, que serviría a Santos Ecay para redactar su comunicación a la

1995. Queremos expresar nuestra gratitud a D. José María Blázquez, que nos allanó el camino para el manejo de esos fondos y respaldó nuestro trabajo; estamos en deuda también con $\mathrm{D}^{\mathrm{a}} \mathrm{M}^{\mathrm{a}} \mathrm{Paz}$ García-Bellido, con quien hemos mantenido fructíferas conversaciones sobre el tema de estas páginas.

${ }^{2}$ El informe de Sanguino se convertiría en artículo esta vez bajo la firma de J. Santos y Ecay, «Antigüedades romanas de Alcuéscar», BRAH 36, 1900, pp. 409-410 (= Revista de Extremadura 2, 1900, pp. 183 ss.), aunque sólo resume los trabajos sin aportar el plano de lo exhumado ni detalle de los descubrimientos.

3 J. Sanguino, «Antigüedades de Las Torrecillas (Alcuéscar)», BRAH 59, 1911, pp. 439-456. 
Academia; a partir de esa fecha continuarían los trabajos de exploración detallados y los resultados obtenidos, con el levantamiento topográfico y las fotografías, serían remitidos a la Academia como informe final en junio de ese mismo año.

\section{LOS TRABAJOS DEL AÑO 1900. UBICACIÓN DEL ENCLAVE}

La finca de Las Torrecillas está ubicada al norte de Alcuéscar a unos $200 \mathrm{~m}$ á la izquierda de un camino de carros, conforme se va hacia Alcuescar para atajar, camino que se desvia de la carretera [de Mérida] al pasar los paradores de Casas de Don Antonio y cruza el monte de arbolado y sale á campo de mieses por entre las que aun se camina un buen trecho antes de dar vista á los cercados de Las Torrecillas ${ }^{4}$. Esta precisa descripción de Sanguino en la memoria final de los trabajos iba acompañada de una serie de indicaciones sobre la proximidad del enclave a la Vía de la Plata, la vía romana que de norte a sur atraviesa estos parajes por el oeste del yacimiento arqueológico. Según consta en esa memoria, el nombre de Las Torrecillas parece provenir de ciertos paredones que aun se levantaban alli hace treinta ó cuarenta años.

\section{DESCRIPCIÓN DE LOS HALLAZGOS}

Con cuantas noticias sobre antigüedades pudieron recoger en el pueblo, los miembros de la delegación enviada a Alcuéscar hicieron un pequeño inventario que se adjuntó al informe, y que contiene referencias no sólo de Las Torrecillas sino también de sus alrededores y algún hallazgo casual en el casco urbano. Afortunadamente en el inventario se especifica claramente la procedencia de unos y otros objetos, por lo que no existe posible confusión al adscribir al enclave las piezas.

En los trabajos agrícolas que dieron lugar a la inspección arqueológica aparecieron sillares de granito, descubriéndose cimientos de edificios, pavimentos, baldosas de mármol, fragmentos de estatuas, objetos varios $y$, en fin, un sótano. Junto a ellos se da cuenta del hallazgo de varias monedas que los comisionados aún pudieron ver y adquirir (vid. infra).

Entre los restos dispersos que aparecieron en el enclave o reaprovechados en los tapiales próximos

4 Todas las citas en cursiva corresponden a la «Memoria» de 1900 . se encontraron varias piezas de piedra pertenecientes a una conducción de agua; dichos elementos, con un canal semicircular tallado sobre bloques regulares de piedra, medían $1 \mathrm{~m}$ de longitud, con 20 $\mathrm{cm}$ de base y otros tantos de altura. Sin duda se trataba de los elementos de la conducción que traía el agua hacia el recinto que constituyó el objeto central de las exploraciones ${ }^{5}$.

El área en donde se produjeron los hallazgos se extendía hacia el Este en dirección al valle del Chamorro, cruzado por el arroyo del Aceite que nace en Alcuéscar y desemboca en el río Ayuela. Pero también se realizaron descubrimientos casuales en las fincas aledañas, lo que demuestra que el enclave arqueológico tenía unas considerables proporciones y excedía con mucho el lugar en el que se centraron los trabajos.

Según contaron los vecinos a los miembros de la comisión, hace ocho o diez años se halló en uno de estos cercados un pie de mármol unido á la basa que tuviera la estatua de modelado bastante bueno, que conserva una señora del pueblo; la pieza aún existía en 1900 y pudo fotografiarse para adjuntarla al expediente que se envió a Madrid; se describe como de mármol sacaroideo y es la parte inferior de un relieve con la parte posterior sin rebajar para ser adosado a un lienzo de muro, y en él puede verse el pie derecho desnudo de una figura humana junto a la cual parece reconocerse el prótomo de un delfín; fue P. Paris, en una visita al Museo de Cáceres, en donde se conserva el fragmento, el que la identificó como una Venus, aunque en la bibliografía se viene hablando de la figura como parte de una representación de Diana.

Se alude también en este inventario a una figura de bronce representando a Minerva, con los brazos rotos, cuya altura era de $6,6 \mathrm{~cm}$. Fue descubierta en el casco urbano de Alcuéscar al derribar una pared del palacio de los Comendadores de Alcuéscar, de la orden de Santiago, y también se conserva en el Museo Provincial de Cáceres. El último objeto de esta relación de hallazgos casuales es un fragmento de mano que ase un cuerpo cilíndrico, que figura también en las fotografías enviadas a Madrid y que se conserva en el Museo cacereño. En la publicación de 1911 J. Sanguino añadió una imagen de mármol de Minerva que poseía en Madrid D. Eduardo Hernández-Pacheco y que también procedía de Las Torrecillas.

La descripción de los hallazgos monetarios en el

5 El dibujo de uno de ellos figura en la mencionada «Memoria» de 1900 y fue publicado por Sanguino más tarde (cit. n. 3 , p. 442) 
informe de la Comisión de Monumentos de Cáceres constituye un ejemplo de minuciosidad no sólo por la precisa localización de los descubrimientos, sino por los dibujos que acompañan la Memoria, que permiten hoy, casi cien años después, una identificación de los tipos con mínimos márgenes de error. Sanguino y sus compañeros, en ausencia de repertorios de clasificación, se limitaron a dibujar con detalle los hallazgos, por lo que nuestras estimaciones dependen de la fidelidad en el registro de las leyendas o marcas de cecas. Todas las monedas proceden de tres enclaves: el propio lugar de Las Torrecillas, las inmediaciones de la calzada romana en palabras de los descubridores y Los Frontones, junto al puerto de las Herrerías.

\section{A. Las Torrecillas}

A1. Vespasiano. Denario. Roma. 69 d.C.

Anv.: [imp caesar] VESPASIANVS AVG.

Rev.: [cos iter tr pot].

$18 \mathrm{~mm}$

RIC II 6.

A2. Claudio II. Radiado. Ceca no determinable. 268-270 d.C.

Anv.: IMP CLAVDIV[s aug]. Cabeza radiada a derecha.

$20 \mathrm{~mm}$

Rev.: Ilegible.

A3. Constantino. Follis. Roma, of. $3^{\text {a }}$ 316-317 d.C.

Anv.: IMP CONSTANTINVS [p $\mathrm{f}$ aug].

Rev.: SOLI INVICTO COMITI.

Exergo: RT

$21 \mathrm{~mm}$

Tipo RIC VII 57.

\section{B. Calzada romana}

B1. Denario republicano. Roma. 137 a.C.

Anv.: Cabeza galeada a derecha.

Rev.: [fo]STLV[s]. Loba con gemelos bajo ficus Ruminalis.

$19 \mathrm{~mm}$

RRC 235.1.

B2. Julia Domna. Dupondio o As. Roma. 196-211 d.C.

Anv.: IVLIA AVGV[sta].

Rev.: VENVS-VICTRIX, S C.

$18 \mathrm{~mm}$

RIC IV.1, 888-890.
C. Los Frontones, junto al puerto de las Herrerías

C1. Marco Aurelio. Denario. 161-180 d.C

Anv.: [-] - TONINVS AVG. Busto drapeado y diademado a derecha.

Rev.: [-]NVS. Figura en pie sosteniendo globo y labarum.

C2. Constantino. Follis. Tréveris, of. ? 313-317

d.C.

Anv.: [imp const]ANTINVS [p f] AVG.

Rev.: SO[li inuicto comiti].

Exergo: $\frac{T \mid F}{[---]}$

RIC VII, $\mathrm{n}^{\circ}$ no determinable.

C3 - C5. Tres bronces, probablemente ases o semises, en los que no puede reconocerse nada.

\section{EL HYPOCAUSTUM Y LOS HALLAZGOS EN SU INTERIOR}

El descubrimiento más espectacular en Las Torrecillas y el que verdaderamente desató el interés por el enclave fue un hypocaustum en perfecto estado de conservación, con el pavimento superior intacto, que se rompió ligeramente en uno de sus costados y que permitió localizar lo que en los informes manuscritos se denomina el subterráneo y en la publicación el sótano ${ }^{6}$. El hecho de que formara parte de la Comisión enviada a Alcuéscar el arquitecto provincial D. Emilio $\mathrm{M}^{\mathrm{a}}$ Rodríguez permitió la realización de un preciso plano de la estructura ${ }^{7}$ y una descripción técnica de lo conservado.

El hypocaustum estaba tallado en la roca natural por sus caras septentrional y oriental, habiéndose cerrado las otras dos de obra para crear la cámara. Las dimensiones internas del recinto eran $5,70 \mathrm{~m}$ $(\mathrm{N}-\mathrm{S}) \times 6,50 \mathrm{~m}(\mathrm{E}-\mathrm{W})$. En el costado meridional los trabajos agrícolas provocaron la rotura parcial del muro (fig. 1) y se abrió un agujero por el que se entró para realizar el plano y valorar la construcción, ya que en todo momento se hace constar que el suelo de la estancia se conservaba intacto.

El hypocaustum estaba formado por cinco galerías orientadas de Este a Oeste, cada una de las cuales disponía de nueve arcos de medio punto en ladrillo; estos arcos apoyaban sobre pilares de escasa

\footnotetext{
6 Ibid., pp. 446 ss.

7 Inédito. Se conserva en el expediente citado en la Real Academia de la Historia; vid. aquí nuestra figura 1.
} 
altura formados por tres o cuatro ladrillos cada uno. El vano de cada una de las cinco galerías medía 50 $\mathrm{cm}$ de altura, mientras que la distancia entre cada una de las nueve series de arcos oscilaba entre los 30 y $\operatorname{los} 45 \mathrm{~cm}$.

Uno de los puntos de mayor interés del plano y la descripción del recinto es el relativo al pavimento soportado por los arcos. Según la descripción que debe ser de Rodríguez por la precisa valoración arquitectónica - los arcos estaban ligados por lajas de pizarra verticales que iban de la enjuta de unos arcos á la de sus fronteros, muchas rotas; sobre los arcos se observaba la existencia de grandes baldosas cuadradas, tal vez de $50 \mathrm{~cm}$ de lado y $7 \mathrm{~cm}$ de grueso, que deben corresponder a los ladrillos bipedales que sostenían el suelo de la habitación superior, colocado inmediatamente encima.

La estructura de este suelo, según la descripción del informe citado, estaba constituida por tres capas que, de abajo a arriba, los miembros de la comisión describen como suelo de hormigón, capa de tierra y argamasa; dado que en el informe se alude al hallazgo de algunos fragmentos de argamasa con teselas blancas, no cabe duda de que estamos ante un pavimento musivo colocado sobre las suspensurae de arcos, máxime si consideramos que la descripción que se hace de la argamasa superior sugiere que lo hallado fue una cama de mosaico en un signinum bastante alterado.

Dentro de la cámara del hypocaustum se hallaron un cráneo de toro, tres calaveras humanas, huesos, una moneda perdida, un cazo de hierro, un cuchillo y lo que, a tenor del dibujo que consta en el informe y de la descripción publicada por Sanguino ${ }^{8}$, es una clavija de concameratio perteneciente sin duda alguna a la cámara de aire de la habitación superior ${ }^{9}$. No se cita en la «Memoria», pero sí en la publicación ${ }^{10}$, un capitel del que los comisionados adjuntan fotografías pese a que no fue encontrado durante sus pesquisas sino que había aparecido con anterioridad ${ }^{11}$.

El cuchillo al que se alude en el informe ${ }^{12}$, y que figura en una de las fotografías que lo acompañan ${ }^{13}$, era de hierro y medía $23,5 \mathrm{~cm}$ de longitud; se trata de un cuchillo de dorso recto y hoja curva con escotadura superior separando el espigón del man-

\footnotetext{
8 J. Sanguino, cit. (n. 3), p. 449.

9 Sobre este tipo de piezas en Hispania, cfr. R. Sanz Gamo, «Algunos materiales romanos utilizados en la construcción de las concamerationes», Oretum 3, 1987, pp. 223236.

10 J. Sanguino, cit. (n. 3), p. 451.

$"$ Ibid., p. 451. En la fotografía $n^{\circ} 1$ del manuscrito, junto a los objetos metálicos, aparece el citado capitel, que se conserva hoy en el Museo de Cáceres.

12 Ibid., p. 448

13 Fotografía $n^{\circ} 1$ del manuscrito.
}

go, que se ajusta al modelo habitualmente definido como «tipo Simancas» ${ }^{14}$. La escotadura superior que separa el espigón del mango en este ejemplar encuentra además su paralelo en algunos hallazgos de la Meseta norte como son las necrópolis de San Miguel del Arroyo (Burgos), Nuez de Abajo (Burgos), Fuentespreadas (Zamora) ${ }^{15}$, Aguilar de Anguita ${ }^{16}$ y Mucientes (Valladolid) ${ }^{17}$. El que los informantes llaman cazo de hierro ${ }^{18}$ medía $42 \mathrm{~cm}$ de longitud, con un recipiente de $12,3 \mathrm{~cm}$ de diámetro y parece tratarse de un simpulum ${ }^{19}$, aunque no sea habitual encontrarlos en hierro.

\section{LAS TORRECILLAS Y SU IDENTIFICACIÓN HISTÓRICA}

La localidad de Alcuéscar ha saltado a la actualidad científica en los últimos años gracias a la excavación y restauración del templo de Santa $\mathrm{Lu}$ cía del Trampal ${ }^{20}$, en donde ha sido hallado un riquísimo conjunto epigráfico que vincula el área al culto de Ataecina y que sugiere la ubicación en las proximidades del antiguo núcleo de Turobriga.

$\mathrm{Al}$ estudiar ese rico conjunto epigráfico ${ }^{21}$ ya su-

14 El hallazgo que da nombre al grupo puede verse en $\mathrm{S}$. Rivera, «La necrópolis visigoda de Simancas», BSEAA 13, 1940 , pp. 7 ss.; sobre los cuchillos y sus lugares de hallazgo, vid. P. de Palol, «Cuchillo hispanorromano del siglo IV de J.C.», BSEAA 30, 1964, pp. 67-102 y L. Caballero, La necrópolis tardorromana de Fuentespreadas (Zamora). Un asentamiento en el valle del Duero. EAE 80, Madrid 1974, p. 58, n. 85 s., fig. 15 y 47 .

15 L. Caballero, cit. (n. 14), p. 100, fig. 15 B y C

16 L. Caballero, cit. (n. 14), p. 59, fig. 14, 2; J. SánchezLafuente, «Aportaciones al estudio del campamento romano de La Cerca (Aguilar de Anguita, Guadalajara)», Wad-al-Hayara 6,1979 , p. 79 y fig. 2 .

17 G. Delibes, «Hallazgos tardorromanos en Mucientes (Valladolid)», BSEAA 36, 1970, p. 462, fig. 1.

${ }^{18}$ Fotografía $\mathrm{n}^{\circ} 1$ del manuscrito.

19 Identificado correctamente por J. Sanguino, cit. (n. 3), p. 449.

${ }^{20}$ Las referencias bibliográficas sobre el templo y sus alrededores son numerosas; $c f r$. principalmente L. Caballero y J. Rosco, «Iglesia visigoda de Santa Lucía del Trampal, Alcuéscar (prov. Cáceres). Primera campaña de trabajos arqueológicos 1983-84», Extremadura Arqueológica 1, 1988, pp. 231-249; L. Caballero, «Santa Lucía del Trampal, Alcuéscar (Cáceres). Una nueva iglesia visigoda», Información Cultural. Ministerio de Cultura 75, 1989, pp. 12 ss.; L. Caballero, A. Almagro, A. Madroñero y A. Granda, «La iglesia de época visigoda de Santa Lucía del Trampal. Alcuéscar (Cáceres)», Extremadura arqueológica 2, 1991, pp. 497-523. Una relación exhaustiva puede verse J. M. Abascal, «Las inscripciones latinas de Santa Lucía del Trampal (Alcuéscar, Cáceres) y el culto de Ataecina en Hispania», $A E A 68,1995$, pp. 31-105, especialmente p. 32, nota 2 .

${ }_{21}$ J. M. Abascal, cit. (n. 20), p. 75; id., «La dea domina sancta Turibrigensis Ataecina y las nuevas evidencias epigráficas de Alcuéscar (Cáceres)», en prensa en las actas del coloquio de Sintra 1995 sobre cultos prerromanos. 


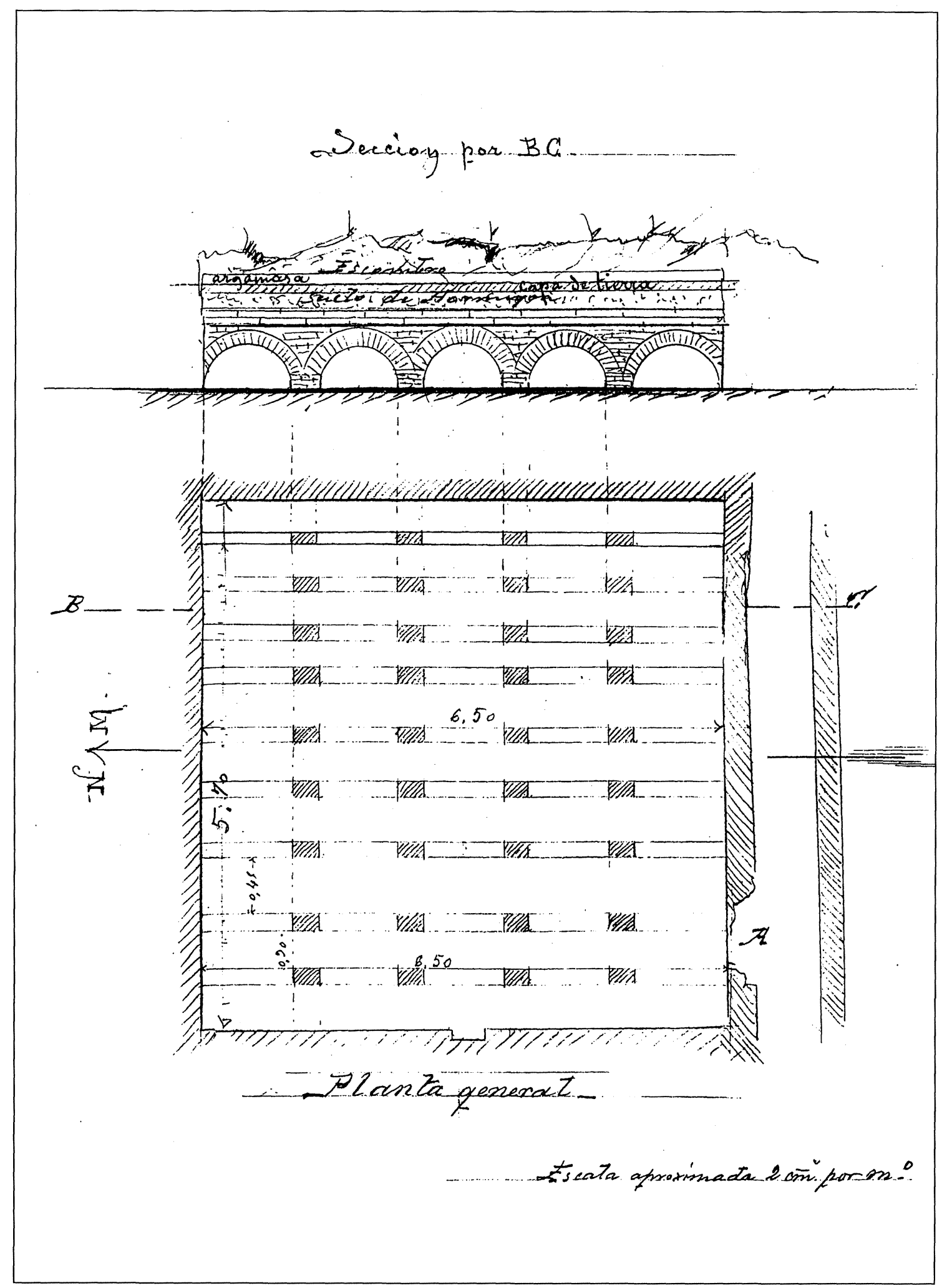

Figura 1.- Plano del hypocaustum de Las Torrecillas. 
gerimos que las estelas funerarias que servían de umbrales en el templo tenían una gran similitud con otra, conservada en Alcuéscar, que procedía de Las Torrecillas, lo que hacía probable que todo el conjunto hubiera sido acarreado desde allí para la construcción del templo.

El epígrafe de Las Torrecillas, que servía de asiento en un molino de aceite en Alcuéscar, es una estela de granito con cabecera semicircular ${ }^{22}$, de algo más de un metro de altura, con una doble cartela que contiene dos textos funerarios y un creciente lunar superior. Las semejanzas formales entre este ejemplar y las estelas de Santa Lucía son enormes incluso en sus dimensiones; la única diferencia sensible, la altura, no constituye un obstáculo, ya que en los ejemplares de Santa Lucía el texto ocupa sólo la parte superior del monumento, mientras el resto del soporte se encuentra sin trabajar, y en la estela de Las Torrecillas la parte inferior se ha perdido, por lo que no es descartable que nos encontremos ante un monumento del mismo tipo.

La Memoria de los trabajos llevados a cabo por Juan Sanguino y sus colaboradores en Las Torrecillas demuestra que el enclave, muy superior en extensión a lo que ellos conocieron, fue un asentamiento romano de envergadura con una dilatada existencia. Los hallazgos numismáticos aseguran la pervivencia de la ocupación durante todo el Principado e incluso en época tardorromana. Desgraciadamente no podemos calibrar el alcance real del área arqueológica por la ausencia de trabajos de excavación en el yacimiento; sin embargo, la prospección de la zona demuestra la importancia de la ocupación humana en época romana ${ }^{23}$ y realza el papel de establecimiento en la organización del hábitat en esta zona septentrional del territorium de Emerita Augusta.

El interés de Las Torrecillas estriba en la combinación de huellas de la vida cotidiana y de evidencias de las prácticas funerarias. Éstas últimas, básicamente las inscripciones, no pudieron ser empleadas en el enclave como materiales de construcción, puesto que ésta sería su función definitiva en Santa Lucía, por lo que hay que imaginar que proceden del expolio de una necrópolis. Tal área de enterramientos combinada con la pequeña parte del conjunto exhumada por Sanguino avala, a falta de hipótesis más convincentes, la idea de que en el lugar existió un núcleo de población; este núcleo, si hacemos caso a las evidencias epigráficas de Santa Lucía, bien pudo ser la Turibriga o Turobriga que tuvo como diosa tutelar a Ataecina, sin que ello presuponga la existencia de un núcleo urbano, pudiendo tratarse únicamente de un centro religioso con una pequeña aldea anexa.
${ }_{22}$ A. González Cordero et alii, «Nuevas aportaciones a la epigrafía de Extremadura», Studia Zamorensia $6,1985, \mathrm{n}^{\circ} 7$, pp. 293-295, lám. 6 (= HEp 1, 152); V. Soria Sánchez, «Inscripciones romanas en Extremadura», XVI Coloquios Históricos de Extremadura, Trujillo 1987, p. 26, $\mathrm{n}^{\circ} 2$; id., Aguas Vivas 8,1988 , III, $\mathrm{n}^{\circ} 2$.
${ }^{23}$ Dato que debemos a la amabilidad del Prof. Enrique Cerrillo. 